\title{
REMARKS ON TOPOLOGICAL ALGEBRAS
}

\author{
A. Beilinson \\ University of Chicago \\ To Victor Ginzburg on his 50th birthday
}

The note complements "topological" aspects of the chiral algebras story from $[\mathrm{BD}]$. In its first section (which has a whiff of $[\mathrm{G}]$ in it) we show that the basic chiral algebra format (chiral operations, etc.) has a precise analog in the setting of topological linear algebra. This provides, in particular, a natural explanation of the passage from chiral to topological algebras from $[\mathrm{BD}] 3.6$. The second section is a brief discussion, in the spirit of [BD] 3.9, of topological algebras similar to rings of chiral differential operators. We also correct some errors from [BD].

The writing was prompted by a talk of D. Gaitsgory at the end of 2004; I am grateful to him for helpful discussions.

\section{TOPOLOGICAL TENSOR PRODUCTS AND TOPOLOGICAL ALGEBRAS.}

1.1. The tensor products. For us, as in $[\mathrm{BD}]$ 3.6.1, "topological vector space" is a $k$-vector space equipped with a linear topology assumed (unless stated explicitly otherwise) to be complete and separated. The category of topological vector spaces is denoted by $\mathcal{T}$ op. This is an additive Karoubian $k$-category.

The category $\mathcal{T} o p$ is quasi-abelian in the sense of [S]. In particular, it is naturally an exact category: the admissible monomorphisms are closed embeddings, the admissible epimorphisms are open surjections.

Remark. If the topology of $B \in \mathcal{T}$ op admits a countable base, then every short exact sequence $0 \rightarrow A \rightarrow B \rightarrow C \rightarrow 0$ is split exact, i.e., $B$ is isomorphic to $A \oplus C$.

Let $\left\{V_{i}\right\}_{i \in I}$ be a finite non-empty collection of topological vector spaces. Consider the tensor product $\otimes V_{i}$. This is an abstract vector space; it carries several natural linear topologies. Namely:

(a) The $*$ topology is formed by all subspaces $Q$ of $\otimes V_{i}$ which satisfy the following property: for every subset $J \subset I$ and vector $v \in \underset{i \in I \backslash J}{\otimes} V_{i}$ there exist open subspaces $P_{j} \subset V_{j}, j \in J$, such that $Q \supset\left(\otimes P_{j}\right) \otimes v$. Let $\otimes^{*} V_{i}$ be the corresponding completion. Then for any topological space $F$ a continuous morphism $\otimes^{*} V_{i} \rightarrow F$ is the same as a continuous polylinear map $\times V_{i} \rightarrow F$.

(b) The ! topology has base formed by vector subspaces $\sum_{i \in I} P_{i} \otimes\left(\underset{i^{\prime} \in I \backslash\{i\}}{\otimes} V_{i^{\prime}}\right)$ where $P_{i} \subset V_{i}$ are any open subspaces. The corresponding completion is denoted by $\otimes ! V_{i}$. Thus $\otimes ! V_{i}=\underline{\lim } \otimes\left(V_{i} / P_{i}\right)$.

Notice that $[\mathrm{BD}]$ (in particular, in $[\mathrm{BD}] 3.6 .1$ ) used the notation $\hat{\otimes} V_{i}$; the reason for the change of notation will become clear later.

(c) Suppose we have a linear order $\tau$ on $I$, i.e., an identification $\{1, \ldots, n\} \stackrel{\sim}{\longrightarrow} I$. It defines on $\otimes V_{i}$ the $\tau$ topology formed by all subspaces $Q$ which satisfy the following 
property: for every $a \in\{1, \ldots, n\}$ and vector $v \in V_{\tau(a+1)} \otimes \ldots \otimes V_{\tau(n)}$ there exists an open subspace $P_{a} \subset V_{\tau(a)}$ such that $Q \supset V_{\tau(1)} \otimes \ldots V_{\tau(a-1)} \otimes P_{a} \otimes v$. The corresponding completion is denoted by $\vec{\otimes}^{\tau} V_{i}=V_{\tau(1)} \vec{\otimes} \ldots \vec{\otimes} V_{\tau(n)}$.

We refer to morphisms $\otimes V_{i} \rightarrow F$ which are continuous with respect to the ! topology, i.e., morphisms $\otimes ! V_{i} \rightarrow F$, as $\otimes$ !-continuous polylinear maps. Same for the other tensor products. ${ }^{1}$

Remarks. (i) Let $U, V$ be topological vector spaces. Suppose first that $V$ is discrete. Then $U \otimes^{*} V=U \vec{\otimes} V$ is equal to $U \otimes V$ equipped with the ind-topology. Precisely, write $V=\underline{\varliminf} V_{\alpha}$ where $V_{\alpha}$ runs the directed set of finite-dimensional subspaces of $V$; then $U \otimes V=\underline{\varliminf_{1 m}} U \otimes V_{\alpha}$, and each $U \otimes V_{\alpha}$ carries an evident topology (as the product of finitely many copies of $U$ ), which defines the said inductive limit topology on $U \otimes V$.

If $V$ is arbitrary, then $U \vec{\otimes} V=\lim U \vec{\otimes}(V / P)$, the projective limit is taken along the set of all open subspaces $P \subset V$.

(ii) Suppose we have $A \in \mathcal{T}$ op and an associative bilinear product $\cdot: A \otimes A \rightarrow A$. Then $\cdot$ is $\otimes^{*}$-continuous if and only if the product map $A \times A \rightarrow A$ is continuous; it is $\vec{\otimes}$-continuous if and only if it is continuous and the open left ideals form a base of the topology of $A ;^{2}$ and it is $\otimes$-continuous if and only if the open two-sided ideals form a base of the topology.

The tensor products $\otimes^{*}$ and $\otimes^{!}$are commutative and associative; they define tensor structures on $\mathcal{T} o p$ which we denote by $\mathcal{T}_{o p}$ ! and $\mathcal{T}_{o p} p^{*}$. The tensor product $\vec{\otimes}$ is associative but not commutative; it defines a monoidal structure on $\mathcal{T}$ op.

Exercise. The tensor products are exact.

The $*$ topology on $\otimes V_{i}$ is stronger than each of the $\tau$ topologies, which in turn are stronger than the ! topology, so the identity map for $\otimes V_{i}$ gives rise to the natural continuous morphisms

$$
\otimes^{*} V_{i} \rightarrow \vec{\otimes}^{\tau} V_{i} \rightarrow \otimes^{!} V_{i}
$$

Lemma. The * topology is equal to the supremum of the $\tau$ topologies for all linear orders $\tau$ on I. The! topology is the infimum of the $\tau$ topologies. I.e., the arrows in (1.1.1) are, respectively, admissible mono- and epimorphism.

Corollary. For any pair $U, V$ of topological vector spaces the short sequence

$$
0 \rightarrow U \otimes^{*} V \rightarrow U \vec{\otimes} V \oplus V \vec{\otimes} U \rightarrow U \otimes ! V \rightarrow 0
$$

where the left arrow comes from the diagonal map and the right one from the difference of the projections, is exact.

1.2. Example. Suppose $F, G$ are Tate vector spaces (see e.g. [BD] 2.7.7, [D] 3.1). Let $F^{*}$ be the dual Tate space to $F$, so $F^{*} \otimes G$ is the vector space of all continuous

\footnotetext{
${ }^{1}$ So $\otimes^{*}$-continuous polylinear maps are the same as continuous polylinear maps.

${ }^{2}$ Or, equivalently, the open left ideals form a base of the topology and for every $r \in A$ either of endomorphisms $a \mapsto a r$ or $a \mapsto a r-r a$ of $A$ is continuous.
} 
maps $F \rightarrow G$ with finite-dimensional image. Then $F^{*} \otimes ! G$ identifies naturally with the space $\operatorname{Hom}(F, G)$ of all continuous linear maps $F \rightarrow G, F^{*} \vec{\otimes} G$ with the space $\operatorname{Hom}_{c}(F, G)$ of maps having bounded image, $G \vec{\otimes} F^{*}$ with the $\operatorname{space}^{H_{0}}(F, G)$ of maps having open kernel, and $F^{*} \otimes^{*} G$ with $\operatorname{Hom}_{f}(F, G):=\operatorname{Hom}_{c}(F, G) \cap$ $\operatorname{Hom}_{d}(F, G)$. So the vector spaces $\operatorname{Hom}(F, G), \operatorname{Hom}_{c}(F, G), \operatorname{Hom}_{d}(F, G), \operatorname{Hom}_{f}(F, G)$ carry natural topologies, and (1.1.2) yields a short exact sequence in $\mathcal{T} o p$

$$
0 \rightarrow \operatorname{Hom}_{f}(F, G) \rightarrow \operatorname{Hom}_{c}(F, G) \oplus \operatorname{Hom}_{d}(F, G) \rightarrow \operatorname{Hom}(F, G) \rightarrow 0 .
$$

Let us describe these topological vector spaces explicitly. Choose decompositions $F=F_{c} \oplus F_{d}, G=G_{c} \oplus G_{d}$ where $F_{c}, G_{c}$ are c-lattices, $F_{d}, G_{d}$ are d-lattices. Consider the corresponding decomposition of the Hom spaces into the sum of the four subspaces. One has:

- $\operatorname{Hom}\left(F_{c}, G_{d}\right)=\operatorname{Hom}_{c}\left(F_{c}, G_{d}\right)=\operatorname{Hom}_{d}\left(F_{c}, G_{d}\right)=\operatorname{Hom}_{f}\left(F_{c}, G_{d}\right)$ : this is a discrete vector space;

- $\operatorname{Hom}\left(F_{d}, G_{c}\right)=\operatorname{Hom}_{c}\left(F_{d}, G_{c}\right)=\operatorname{Hom}_{d}\left(F_{d}, G_{c}\right)=\operatorname{Hom}_{f}\left(F_{d}, G_{c}\right)$ : this is a compact vector space;

- $\operatorname{Hom}\left(F_{c}, G_{c}\right)=\operatorname{Hom}_{c}\left(F_{c}, G_{c}\right)$ and $\operatorname{Hom}_{d}\left(F_{c}, G_{c}\right)=\operatorname{Hom}_{f}\left(F_{c}, G_{c}\right)$; the latter topological vector space equals $G_{c} \otimes F_{c}^{*}$ equipped with the ind-topology;

- $\operatorname{Hom}\left(F_{d}, G_{d}\right)=\operatorname{Hom}_{d}\left(F_{d}, G_{d}\right)$ and $\operatorname{Hom}_{c}\left(F_{d}, G_{d}\right)=\operatorname{Hom}_{f}\left(F_{d}, G_{d}\right)$; the latter topological vector space equals $F_{d}^{*} \otimes G_{d}$ equipped with the ind-topology.

A correction to $[\mathrm{BD}]$ 2.7.7. In loc. cit., certain topologies on the Hom spaces were considered. The topology on $\operatorname{Hom}(F, G)$ coincides with the above one, but those on $\operatorname{Hom}_{c}, \operatorname{Hom}_{d}$ and $\mathrm{Hom}_{f}$ are stronger than the above topologies, and they are less natural. Precisely, these topologies differ at terms $\operatorname{Hom}_{d}\left(F_{c}, G_{c}\right)=\operatorname{Hom}_{f}\left(F_{c}, G_{c}\right)$ and $\operatorname{Hom}_{c}\left(F_{d}, G_{d}\right)=\operatorname{Hom}_{f}\left(F_{d}, G_{d}\right)$ which are considered in [BD] 2.7.7 as discrete vector spaces. Exact sequence (1.2) coincides with [BD] (2.7.7.1) (up to signs); it is strongly compatible with either of the topologies. These topologies played an auxiliary role: they were used to define (in [BD] 2.7.8) the topology on the Tate extension $\mathfrak{g l}(F)^{b}$ as the quotient topology for the canonical surjective map $\operatorname{End}_{c}(F) \oplus \operatorname{End}_{d}(F) \rightarrow \mathfrak{g l}(F)^{b}$. Replacing them by the present topologies does not change the quotient topology on $\mathfrak{g l}(F)^{b}$. We suggest to discard the topologies on $\operatorname{Hom}_{c}, \operatorname{Hom}_{d}, \operatorname{Hom}_{f}$ from [BD] 2.7.7, and replace them by those defined above.

1.3. The chiral operations. As in [BD] 1.1.3, the ! and $*$ tensor structures on $\mathcal{T}_{o p}$ can be seen as pseudo-tensor structures with the spaces of operations $P_{I}^{!}\left(\left\{V_{i}\right\}, F\right):=\operatorname{Hom}\left(\otimes^{!} V_{i}, F\right)$ and $P_{I}^{*}\left(\left\{V_{i}\right\}, F\right):=\operatorname{Hom}\left(\otimes^{*} V_{i}, F\right)$.

The tensor product $\vec{\otimes}$ is non-commutative, so the definition of the corresponding pseudo-tensor structure requires an appropriate induction:

$A$ digression: Let $\Sigma$ be the linear orders operad (see [BD] 1.1.4). Suppose we have a $k$-category $\mathcal{T}$ equipped with an associative tensor product (i.e., a monoidal structure) $\otimes$. For a finite non-empty collection of objects $\left\{V_{i}\right\}_{i \in I}$ and a linear order $\tau \in \Sigma_{I}$, i.e., $\tau:\{1, \ldots, n\} \stackrel{\sim}{\rightarrow} I$, we set $\otimes^{\tau} V_{i}:=V_{\tau(1)} \otimes \ldots \otimes V_{\tau(n)}$. We define the $i n$ duced pseudo-tensor structure on $\mathcal{T}$ by formula $P_{I}^{\otimes}\left(\left\{V_{i}\right\}, F\right):=\underset{\tau \in \Sigma_{I}}{\oplus} P_{I}^{\otimes}\left(\left\{V_{i}\right\}, F\right)^{\tau}$ 
where $P_{I}^{\otimes}\left(\left\{V_{i}\right\}, F\right)^{\tau}:=\operatorname{Hom}\left(\otimes^{\tau} V_{i}, F\right)$. The composition of operations is defined in the evident way so that the $\Sigma$-grading is compatible with the composition.

If $\otimes$ is actually commutative, then $P_{I}^{\otimes}\left(\left\{V_{i}\right\}, F\right)=\operatorname{Hom}\left(\otimes V_{i}, F\right) \otimes \mathcal{A} s s_{I}$ where $\mathcal{A} s s=k[\Sigma]$ is the associative algebras operad (see [BD] 1.1.7). The construction is functorial with respect to natural morphisms between $\otimes$ 's.

Applying this to $\mathcal{T}$ op and $\vec{\otimes}$, we get the chiral operations $P^{c h}:=P^{\vec{\otimes}}$; those from the $\tau$-component of $P^{c h}$ are called chiral $\tau^{\tau}$ operations. They define the chiral pseudotensor structure $\mathcal{T}_{o} p^{c h}$ on $\mathcal{T}$ op. This pseudo-tensor structure is representable (see [BD] 1.1.3) with the pseudo-tensor product $\otimes^{c h} V_{i}=\underset{\tau \in \Sigma_{I}}{\oplus} \vec{\otimes}^{\tau} V_{i}$.

We see that (1.1.1) gives rise to natural transformations

$$
P_{I}^{!}\left(\left\{V_{i}\right\}, F\right) \otimes \mathcal{A} s s_{I} \rightarrow P_{I}^{c h}\left(\left\{V_{i}\right\}, F\right) \rightarrow P_{I}^{*}\left(\left\{V_{i}\right\}, F\right) \otimes \mathcal{A} s s_{I}
$$

compatible with the composition of operations, i.e., the identity functor of $\mathcal{T}$ op lifts naturally to pseudo-tensor functors $\mathcal{T} o p^{!} \otimes \mathcal{A} s s \rightarrow \mathcal{T}_{o p}{ }^{c h} \rightarrow \mathcal{T}_{o p}{ }^{*} \otimes \mathcal{A} s s$.

Remarks. (i) The arrows in (1.3.1) are injective. If all the $V_{i}$ are discrete, then they are isomorphisms.

(ii) The endofunctor of $\mathcal{T}$ op which assigns to a topological vector space $V$ same $V$ considered as a discrete space lifts in the obvious manner to a faithful pseudo-tensor endofunctor for all the above pseudo-tensor structures.

Composing (1.3.1) from the left and right with the standard embedding $\mathcal{L} i e \hookrightarrow$ $\mathcal{A} s s$ and projection $\mathcal{A} s s \rightarrow \mathcal{C}$ om,${ }^{3}$ we get natural morphisms (cf. [BD] 3.2.1, 3.2.2)

$$
P_{I}^{!}\left(\left\{V_{i}\right\}, F\right) \otimes \mathcal{L} i e_{I} \rightarrow P_{I}^{c h}\left(\left\{V_{i}\right\}, F\right) \rightarrow P_{I}^{*}\left(\left\{V_{i}\right\}, F\right)
$$

By (1.1.2), in case of two arguments we get an exact sequence

$$
0 \rightarrow P_{2}^{!}(\{U, V\}, F) \otimes \mathcal{L} i e_{2} \rightarrow P_{2}^{c h}(\{U, V\}, F) \rightarrow P_{2}^{*}(\{U, V\}, F)
$$

1.4. Chiral algebras in the topological setting. Let $A$ be a topological vector space. A Liech algebra (or non-unital chiral algebra) structure on $A$ is a Lie bracket $\mu_{A}: A \otimes^{c h} A \rightarrow A$ for the chiral pseudo-tensor structure; we call such $\mu_{A}$ a chiral product on $A$.

Consider the map $P_{2}^{c h}(\{A, A\}, A) \rightarrow \operatorname{Hom}(A \vec{\otimes} A, A)$ which assigns to a binary chiral operation its first component.

Proposition. This map establishes a bijection between the set of chiral products on $A$ and the set of associative products $A \vec{\otimes} A \rightarrow A$.

Proof. Our map yields a bijection between the set of skew-symmetric binary chiral operations and $\operatorname{Hom}(A \vec{\otimes} A, A)$. It remains to check that a skew-symmetric

\footnotetext{
${ }^{3}$ Here $\mathcal{L}$ ie is the Lie algebras operad and $\mathcal{C}$ om is the commutative algebras operad, i.e, the unit $k$-operad.
} 
chiral operation $\mu$ satisfies the Jacobi identity if and only if the corresponding product $A \vec{\otimes} A \rightarrow A$ is associative.

By Remark (ii) from 1.3 it suffices to consider the case of discrete $A$, so now $A$ is a plain vector space. Let $P(A)$ be the operad of polylinear endooperations on $A$, i.e., $P(A)_{I}:=\operatorname{Hom}\left(A^{\otimes I}, A\right)$. By Remark (i) from 1.3 the operad $P^{c h}(A)$ of chiral endooperations of $A$ equals $P(A) \otimes \mathcal{A} s s$.

Let $\cdot{ }_{a s} \in \mathcal{A} s s_{2}$ be the standard associative binary operation. We want to prove that a binary operation $\cdot_{A} \in P(A)_{2}$ is associative if and only if the binary operation $\cdot{ }_{A} \otimes \cdot{ }_{a s}-{ }_{A}^{t} \otimes \cdot{ }_{a s}^{t}$ in $P(A) \otimes \mathcal{A s s}$ is a Lie bracket (here ${ }^{t}$ is the transposition of arguments). Indeed, if $\cdot_{A}$ is associative, then such is $\cdot_{A} \otimes \cdot{ }_{a s}$, hence the commutator $\cdot{ }_{A} \otimes \cdot{ }_{a s}-\cdot_{A}^{t} \otimes \cdot t_{a s}^{t}$ is a Lie bracket. The converse statement is a simple computation left to the reader.

Remark. Here is a reformulation of the proposition in a non-commutative algebraic geometry style. We forget about the topologies. Let $A$ be any vector space. Suppose we have an associative algebra structure on $A$. Then for any test associative algebra $R$ the tensor product $A \otimes R$ is naturally an associative algebra, hence a Lie algebra. In other words, let $\phi_{A}$ be the functor $R \mapsto A \otimes R$ from the category of associative algebras to that of vector spaces; then an associative algebra structure on $A$ yields a Lie algebra structure on $\phi_{A}$ (i.e., a lifting of $\phi_{A}$ to the category of Lie algebras). The claim is that this establishes a bijection between the set of associative algebra structures on $A$ and that of Lie algebra structures on $\phi_{A}$.

For $A \in \mathcal{T}$ op a topological associative algebra structure on $A$ is a continuous associative bilinear product $A \times A \rightarrow A$, i.e., an associative product $A \otimes^{*} A \rightarrow A$. One can sum up the above proposition as follows (the equivalence of (ii), (iii), and (iv) is Remark (ii) in 1.1):

Claim. For $A \in \mathcal{T}$ op the following structures on $A$ are equivalent:

(i) A non-unital chiral algebra structure $\mu_{A}$;

(ii) An associative product $\cdot_{A}: A \vec{\otimes} A \rightarrow A$;

(iii) A topological associative algebra structure such that the open left ideals form a base of the topology of $A$.

(iv) An associative algebra structure such that the open left ideals form a base of the topology of $A$ and the corresponding Lie bracket is continuous.

A correction to $[\mathrm{BD}]$ 3.6.1: In loc. cit. this was recklessly called "topological associative algebra" structure; we suggest to rescind this confusing terminology.

A linguistic comment. The term "chiral" refers to the breaking of symmetry between the right and left movers in physics and is rather awkward in the "purely holomorphic" setting of [BD] (where only one type of movers is present). In the topological setting it looks more suitable for we consider associative products whose left-right asymmetry is enforced by the topology (though now it has to do rather with the time ordrering of physicists).

One often constructs topological chiral algebras using the next corollary: 
Corollary. Let $T$ be an associative algebra equipped with a (not necessary complete) linear topology. Suppose that

- the open left ideals form a base of the topology;

- for every $r$ from a set of associative algebra generators the endomorphisms $t \mapsto[r, t]:=r t-\operatorname{tr}$ of $T$ are continuous.

Then the completion of $T$ is a chiral algebra.

Proof. The endomorphisms $t \mapsto a t$ of $T$ are continuous for any $a \in T$ by the first property, so the second property amounts to continuity of the endomorphisms $t \mapsto t r$ for $r$ from our system of generators, hence they are continuous for every $r \in T$. Thus the completion of $T$ carries the structure from (ii) of the claim.

Suppose we have a topological Lie algebra $L$, i.e., $L$ is a topological vector space equipped with a continuous Lie bracket (i.e., a Lie bracket with respect to $\otimes^{*}$ ). We say that $L$ is a topological Lie* algebra if it satisfies the following technical condition: the open Lie subalgebras form a base of the topology of $L$. Notice that this condition holds automatically if $L$ is a Tate vector space. ${ }^{4}$

Now the second arrow in (1.3.2) transforms any chiral product $\mu_{A}$ into a continuous Lie bracket []$_{A}$. Equivalently, []$_{A}$ is the commutator for the associative product $\cdot A$. By (iii) of the claim, [ ] $A$ is a Lie* algebra structure on $A$.

Our $\mu_{A}$ is said to be commutative if []$_{A}=0$. By by Lemma from 1.1 (and the proposition above) a commutative chiral product amounts to a commutative! algebra structure, i.e., a commutative and associative product $\cdot_{A}: A \otimes ! A \rightarrow A$.

We say that a $\mathrm{Lie}^{c h}$ algebra structure is unital if such is the corresponding associative algebra structure. Such algebras are referred to simply as topological chiral algebras.

For a topological chiral algebra $A$ a discrete $A$-module is a left unital $A$-module $M$ (we consider $A$ as a mere associative algebra now) such that the action $A \times M \rightarrow M$ is continuous (we consider $M$ as a discrete vector space). Equivalently, this is a discrete unital left $A$-module with respect to $\vec{\otimes}$ monoidal structure. Denote by $A \bmod ^{\delta}$ the category of discrete $A$-modules.

Let $\phi: A \bmod ^{\delta} \rightarrow \mathcal{V} e c$ be the forgetful functor (which assigns to a discrete $A$-module its underlying vector space). Then $A$ recovers from $\left(A \bmod ^{\delta}, \phi\right)$ :

Lemma. A equals the topological associative algebra of endomorphisms of $\phi$.

1.5. We denote by $\mathcal{A} s s\left(\mathcal{T} o p^{*}\right)$ the category of topological associative unital algebras, by $\mathcal{C} \mathcal{A}\left(\mathcal{T}_{o p}\right)$ the category of topological chiral algebras, and by $\mathcal{A} s s\left(\mathcal{T}_{o p}{ }^{!}\right)$ that of associative unital algebras with respect to $\otimes$ !.

As we have seen in 1.4 , the above structures on $A \in \mathcal{T}$ op are the same as an associative product $A \otimes A \rightarrow A$ that satisfies stronger and stronger continuity

\footnotetext{
${ }^{4}$ Proof: if $P \subset L$ is a c-lattice, then its normalizer $Q$ is an open Lie subalgebra of $L$, hence $P \cap Q$ is also an open Lie subalgebra.
} 
conditions. Thus we have fully faithful embeddings

$$
\mathcal{A} s s\left(\mathcal{T} o p^{!}\right) \hookrightarrow \mathcal{C} \mathcal{A}(\mathcal{T} o p) \hookrightarrow \mathcal{A} s s\left(\mathcal{T} o p^{*}\right) .
$$

These embeddings admit left adjoint functors

$$
\mathcal{A} s s\left(\mathcal{T} o p^{!}\right) \leftarrow \mathcal{C} \mathcal{A}(\mathcal{T} o p) \leftarrow \mathcal{A} s s\left(\mathcal{T}_{o p}{ }^{*}\right)
$$

Namely, for $A \in \mathcal{A} s s\left(\mathcal{T}_{o p}{ }^{*}\right)$ the corresponding chiral algebra $A^{\text {ch }}$ is the completion of $A$ with respect to the topology whose base is formed by open left ideals, and for $B \in \mathcal{C} \mathcal{A}\left(\mathcal{T}_{o p}\right)$ the corresponding associative! algebra is the completion of $B$ with respect to the topology whose base is formed by open two-sided ideals (see Remark (ii) in 1.1).

Remark. For $B \in \mathcal{A} s s\left(\mathcal{T}_{o p^{*}}\right)$ the category of left unital discrete $A$-modules coincides with $B^{c h} \bmod ^{\delta}$.

The forgetful functor from either of the categories of topological algebras above to $\mathcal{T} o p$ which sends a topological algebra to the underlying topological vector space also admits left adjoint. For $V \in \mathcal{T}$ op the corresponding algebras are denoted by $T^{*} V, T^{c h} V$, and $T^{!} V$. These are completions of the "abstract" tensor algebra $T V:=\underset{n \geq 0}{\oplus} V^{\otimes n}$ with respect to the topology whose base is formed by all subspaces of type $f^{-1}(U)$, where $f: T V \rightarrow A$ is a morphism of associative algebras such that $\left.f\right|_{V}: V \rightarrow A$ is continuous, $A$ is an algebra of our class, and $U \subset A$ is an open subspace.

Remarks. (i) Consider $\mathrm{T}:=\underset{n \geq 0}{\bigoplus} V^{\otimes^{*} n}$. This is a topological vector space (with the inductive limit topology) and an associative algebra, but the product need not be continuous. If $V$ is a Tate space, then the product is continuous if $V$ is either discrete or compact, and not continuous otherwise. ${ }^{5}$

(ii) One obtains $T^{c h} V$ and $T^{!} V$ by applying to $T^{*} V$ the functors from (1.5.2). Certainly, $T^{!} V=\lim T(V / P)$ where $P$ runs the set of an open subspaces of $V$ and $T(V / P)$ is its plain tensor algebra (which is a discrete vector space).

$A$ correction to $[\mathrm{BD}]$ 3.6.1: In loc. cit. there is a wrong claim that $T^{c h} V$ equals the direct sum $\vec{T} V:=k \oplus V \oplus V^{\vec{\otimes} 2} \oplus \ldots$ (equipped with the direct limit topology).

1.6. A topological $\mathcal{D}$-module setting. Let $X$ be our curve and $x \in X$ be a point. We will consider pairs $\left(M, \Xi_{M}\right)$ where $M$ is a $\mathcal{D}$-modules on $X$ and $\Xi_{M}$ is a topology on $M$ at $x$ (see [BD] 2.1.13); such pairs form a $k$-category $\mathcal{M}\left(X, \mathcal{T}_{o} p_{x}\right)$. Denote by $\hat{h}_{x}\left(M, \Xi_{M}\right)$ the completion of the de Rham cohomology stalk $h(M)_{x}$ with respect to our topology (see loc. cit.); we get a functor $\hat{h}_{x}: \mathcal{M}\left(X, \mathcal{T}_{o p}\right) \rightarrow \mathcal{T} o p$.

We will extend $\hat{h}_{x}$ to a pseudo-tensor functor with respect to the !, * and chiral polylinear structures. In order to do this, one needs to explain which operations between $\mathcal{D}$-modules are continuous with respect to our topologies.

(a) * operations (cf. [BD] 2.2.20). Let $\left(M_{i}, \Xi_{M_{i}}\right), i \in I$, be a finite non-empty collection of objects of $\mathcal{M}\left(X, \mathcal{T}_{o p}\right)$.

\footnotetext{
${ }^{5}$ To check the latter assertion, use the next fact (applied to $P:=V^{\otimes} 2, T_{i}:=V^{\otimes^{*} i}$ ): if $P, T_{0}, T_{1}, \ldots \in \mathcal{T} o p$ are non-discrete and $P$ is not Tate, then $\oplus\left(P \otimes^{*} T_{i}\right) \neq P \otimes^{*}\left(\oplus T_{i}\right)$.
} 
Lemma. The $\mathcal{D}_{X^{I}-\text { module }} \nabla M_{i}$ carries a natural topology $\nabla^{*} \Xi_{M_{i}}$ at $\Delta^{(I)}(x)=$ $(x, \ldots, x) \in X^{I}$ such that the corresponding completion of the de Rham cohomology stalk $h\left(\otimes M_{i}\right)_{(x, \ldots, x)}$ equals $\otimes * \hat{h}_{x}\left(M_{i}, \Xi_{M_{i}}\right)$.

Proof. We want to assign in a natural way to every discrete quotient $T$ of $\otimes * \hat{h}_{x}\left(M_{i}, \Xi_{M_{i}}\right)$ a certain quotient of $\otimes M_{i}$ equal to $i_{(x, \ldots, x) *} T$. Since $h_{x}$ commutes with inductive limits, we can assume that each $M_{i}$ is a finitely generated $\mathcal{D}_{X^{-}}$ module. Then $\hat{h}_{x}\left(M_{i}, \Xi_{M_{i}}\right)$ are all compact, so we can assume that $T=\otimes T_{i}$ where $T_{i}$ are discrete (finite-dimensional) quotients of $\hat{h}_{x}\left(M_{i}, \Xi_{M_{i}}\right)$. So $i_{x *} T_{i}$ is a quotient of $M_{i}$, hence $i_{(x, \ldots, x) *} T=\bigotimes i_{x *} T_{i}$ is a quotient of $\otimes M_{i}$, and we are done.

Let $\left(N, \Xi_{N}\right)$ be another object of $\mathcal{M}\left(X, \mathcal{T}_{o p}\right)$. A $*$ operation $\varphi \in P_{I}^{*}\left(\left\{M_{i}\right\}, N\right)$ is said to be continuous with respect to our topologies if $\varphi: \otimes M_{i} \rightarrow \Delta_{*}^{(I)} N$ is continuous with respect to the topologies $\nabla^{*} \Xi_{M_{i}}$ and $\Delta_{*}^{(I)} \Xi_{N}$. The composition of continuous operations is continuous, so they form a pseudo-tensor structure $\mathcal{M}\left(X, \mathcal{T}_{o p} p_{x}\right)^{*}$ on $\mathcal{M}\left(X, \mathcal{T}_{o} p_{x}\right)$. By construction, $\hat{h}_{x}$ lifts to a pseudo-tensor functor

$$
\hat{h}_{x}: \mathcal{M}\left(X, \mathcal{T}_{o p}\right)^{*} \rightarrow \mathcal{T}_{o p}{ }^{*} .
$$

(b) ! operations. From now on we will consider a full subcategory $\mathcal{M}\left(U_{x}, \mathcal{T}_{o} p_{x}\right)$ of $\mathcal{M}\left(X, \mathcal{T}_{o p_{x}}\right)$ formed by those pairs $\left(M, \Xi_{M}\right)$ that $M=j_{x *} j_{x}^{*} M$ where $j_{x}$ is the embedding $U_{x}:=X \backslash\{x\} \hookrightarrow X$. Suppose that our $\left(M_{i}, \Xi_{M_{i}}\right)$ lie in this subcategory.

Lemma. The $\mathcal{D}_{X}$-module $\otimes ! M_{i}$ carries a natural topology $\otimes ! \Xi_{M_{i}}$ at $x$ such that the corresponding completion of $h\left(\otimes ! M_{i}\right)_{x}$ is equal to $\otimes ! \hat{h}_{x}\left(M_{i}, \Xi_{M_{i}}\right)$.

Proof. $\otimes ! \Xi_{M_{i}}$ is the topology with base formed by submodules $\otimes ! P_{i}$ of $\otimes ! M_{i}$ where $P_{i} \subset M_{i}$ are open submodules for $\Xi_{M_{i}}$.

Set $\otimes !\left(M_{i}, \Xi_{M_{i}}\right):=\left(\otimes ! M_{i}, \otimes ! \Xi_{M_{i}}\right)$. This tensor product makes $\mathcal{M}\left(U_{x}, \mathcal{T}_{o} p_{x}\right)$ a tensor category which we denote by $\mathcal{M}\left(U_{x}, \mathcal{T} o p_{x}\right)^{!}$. Its unit object is $j_{x *} \omega_{U_{x}}$ equipped with the topology formed by the open submodule $\omega_{X} \subset j_{x *} \omega_{U_{x}}$. The functor $\hat{h}_{x}$ lifts naturally to a tensor functor

$$
\hat{h}_{x}: \mathcal{M}\left(U_{x}, \mathcal{T}_{o p}\right)^{!} \rightarrow \mathcal{T} o p^{!} .
$$

(c) Chiral operations. Let $\left(M_{i}, \Xi_{M_{i}}\right)$ be, as above, some objects of $\mathcal{M}\left(U_{x}, \mathcal{T}_{o p}\right)$, and $j^{(I)}: U^{(I)} \hookrightarrow X^{I}$ be the complement to the diagonal divisor.

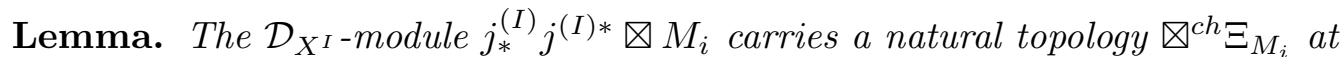
$(x, \ldots, x) \in X^{I}$ such that the corresponding completion of the de Rham cohomology stalk $h\left(j_{*}^{(I)} j^{(I) *} \otimes M_{i}\right)_{(x, \ldots, x)}$ is equal to $\otimes^{c h} \hat{h}_{x}\left(M_{i}, \Xi_{M_{i}}\right)$.

Proof. We proceed by induction by $|I|$. Let us choose for each $i \in I$ a $\Xi_{M_{i}}$ open submodule $P_{i} \subset M_{i}=j_{x *} j_{x}^{*} M_{i}$; set $T_{i}:=h\left(M_{i} / P_{i}\right)_{x}$, so $M_{i} / P_{i}=i_{x *} T_{i}$. Set $I_{i}:=I \backslash\{i\}$. The sequence $\left.0 \rightarrow \bigotimes P_{i} \rightarrow \bigotimes M_{i} \rightarrow \underset{i \in I}{\oplus} \underset{i^{\prime} \in I_{i}}{\otimes} M_{i^{\prime}}\right) \otimes i_{x *} T_{i} \rightarrow 0$ is short exact over $U^{(I)}$. So we have a short exact sequence

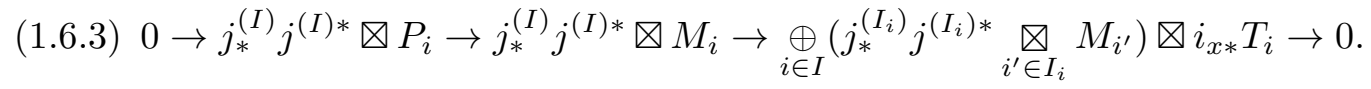


By the induction assumption, for each $i \in I$ the $\mathcal{D}_{X^{I_{i}}}$-module $j_{*}^{\left(I_{i}\right)} j^{\left(I_{i}\right) *} \underset{i^{\prime} \in I_{i}}{\otimes} M_{i^{\prime}}$ carries the topology $\underset{i^{\prime} \in I_{i}}{\bigotimes^{c h}} \Xi_{M_{i^{\prime}}}$ at $(x, \ldots, x)$ with the completed de Rham cohomol-

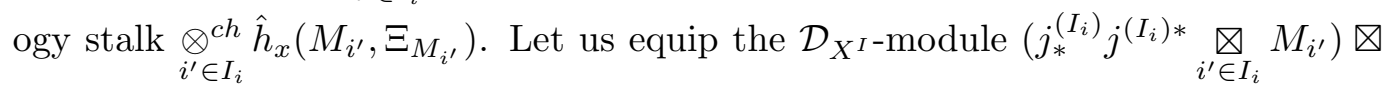

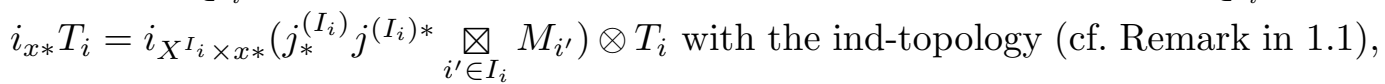
so the completion of its de Rham cohomology stalk equals $\underset{i^{\prime} \in I_{i}}{\otimes^{c h}} \hat{h}_{x}\left(M_{i^{\prime}}, \Xi_{M_{i^{\prime}}}\right) \vec{\otimes} T_{i}$. By (1.6.3), the product of these topologies can be seen as a topology on the quotient module $j_{*}^{(I)} j^{(I) *} \otimes M_{i} / j_{*}^{(I)} j^{(I) *} \otimes P_{i}$ which we denote by $\Xi_{\left(P_{i}\right)}$.

Now our topology $\nabla^{c h} \Xi_{M_{i}}$ is formed by all $\mathcal{D}_{X^{I}}$-submodules $P \subset j_{*}^{(I)} j^{(I) *} \otimes M_{i}$ such that $P$ contains the submodule $j_{*}^{(I)} j^{(I) *} \otimes P_{i}$ for some choice of $\Xi_{M_{i}}$-open $P_{i} \subset M_{i}$ and the image of $P$ in $j_{*}^{(I)} j^{(I) *} \otimes M_{i} / j_{*}^{(I)} j^{(I) *} \otimes P_{i}$ is $\Xi_{\left(P_{i}\right)}$-open.

Let $\left(N, \Xi_{N}\right)$ be another object of $\mathcal{M}\left(U_{x}, \mathcal{T}_{o p}\right)$ and $\varphi \in P_{I}^{c h}\left(\left\{M_{i}\right\}, N\right)$ be a chiral operation. We say that $\varphi$ is continuous with respect to our topologies if $\varphi: j_{*}^{(I)} j^{(I) *} \otimes M_{i} \rightarrow \Delta_{*}^{(I)} N$ is continuous with respect to the topologies $\nabla^{c h} \Xi_{M_{i}}$ and $\Delta_{*}^{(I)} \Xi_{N}$. The composition of continuous operations is continuous, so they form a pseudo-tensor structure $\left.\mathcal{M}\left(X, \mathcal{T}_{o p}\right)\right)^{c h}$ on $\mathcal{M}\left(U_{x}, \mathcal{T}_{o p} p_{x}\right)$. By construction, $\hat{h}_{x}$ lifts to a pseudo-tensor functor

$$
\hat{h}_{x}: \mathcal{M}\left(U_{x}, \mathcal{T}_{o p}\right)^{c h} \rightarrow \mathcal{T}_{o p}{ }^{c h} .
$$

1.7. Example. Let $A$ be a chiral algebra on $U_{x}$. As in [BD] 3.6.4, we denote by $\Xi_{x}^{a s}$ the topology on $j_{x *} A$ at $x$ whose base is formed by all chiral subalgebras of $j_{x *} A$ that coincide with $A$ on $U_{x}$. Set $A_{x}^{a s}:=\hat{h}_{x}\left(j_{x *} A, \Xi_{x}^{a s}\right)$.

Lemma. The chiral product $\mu_{A}$ is $\Xi_{x}^{a s}$-continuous.

Therefore, according to (1.6.4), $A_{x}^{a s}$ is a topological chiral algebra. We leave it to the reader to check that the assocciative product on $A_{x}^{a s}$ coincides with the one defined in $[\mathrm{BD}]$ 3.6.6.

\section{TOPOLOGICAL CDO.}

2.1. The classical limit: from chiral to coisson algebras. Let $A$ be any topological vector space. Below a filtration on $A$ always means an increasing filtration $A_{0} \subset A_{1} \subset \ldots$ by closed vector subspaces of $A$ such that $A_{\infty}:=\cup A_{i}$ is dense in $A$. We have a graded topological vector space gr. $A$ with components $\operatorname{gr}_{i} A:=A_{i} / A_{i-1}$.

The vector space $\oplus \operatorname{gr}_{i} A$ carries a natural topology whose base is formed by subspaces $\oplus\left(P \cap A_{i}\right) /\left(P \cap A_{i-1}\right)$ where $P$ is an open subspace of $A$; we denote by $\widehat{\mathrm{gr}} A$ the completion.

Suppose now that $A$ is a topological chiral algebra and $A$. as above is a ring filtration, i.e., $A_{i} \cdot A_{j} \subset A_{i+j}, 1 \in A_{0}$; we call such $A$. a chiral algebra filtration. Then $\widehat{\mathrm{gr}} A$ is naturally a topological chiral algebra. 
Our $A_{\infty}$ is a subring of $A$. Consider a topology on $A_{\infty}$ formed by all left ideals $I$ in $A_{\infty}$ such that $I \cap A_{i}$ is open in $A_{i}$ for every $i$. For any $r \in A_{\infty}$ the right multiplication endomorphism $a \mapsto a r$ of $A_{\infty}$ is continuous, so (by Corollary in 1.4) the completion $A_{\infty}$ of $A_{\infty}$ with respect to this topology is a topological chiral algebra. The embedding $A_{\infty} \subset A$ extends by continuity to a morphism of topological chiral algebras $A_{\infty} \rightarrow A$.

Definition. A chiral algebra filtration $A$. is admissible if $\underset{A_{\infty}}{\sim} \stackrel{\sim}{\rightarrow} A$, i.e., a closed left ideal $I \subset A$ is open if (and only if) each $I \cap A_{n}$ is open in $A_{n}$.

Example. Consider the algebra $k[[t]]$ equipped with the usual topology. Its chiral algebra filtration $k[[t]]_{n}:=k+k t+\ldots+k t^{n}$ is not admissible.

Below topological coisson algebra means a topological vector space $R$ equipped with a Poisson algebra structure such that the Lie bracket is $\left(\otimes^{*}-\right)$ continuous and the product is $\otimes$-continuous. We also demand $R$ to be a topological Lie* algebra (see 1.4); equivalently, this means that open ideals of the commutative algebra $R$ which are Lie subalgebras form a base of the topology.

Let $A$ be any topological chiral algebra. A chiral algebra filtration on $A$ is said to be commutative if $\operatorname{gr} A$ is a commutative algebra. Then $\widehat{\mathrm{gr}} A$ is a commutative topological chiral algebra, i.e., a commutative! algebra. The usual Poisson bracket on $\operatorname{gr} A$ extends by continuity to a continuous Lie bracket on $\widehat{\mathrm{gr}} A$, which makes $\widehat{\mathrm{gr}} A$ a topological coisson algebra.

2.2. Topological Lie* algebroids. Let $R$ be any (unital) topological commutative! algebra. We denote by $R \bmod !$ the category of unital $R$-modules in the tensor category $\mathcal{T}_{o p} !$. This is a tensor $k$-category with tensor product $\underset{R}{\otimes} !$, and an exact category: a short sequence of $R$-modules is exact if it is exact as a sequence in $\mathcal{T} o p$ (see 1.1). For $M \in R \bmod !$ and an open ideal $I \subset R$ we write $M_{R / I}:=(R / I) \underset{R}{\otimes}{ }_{R} M=$ $M / \overline{I M}$ (here $\overline{I M}$ is the closure of $I M$ ); this is a topological $R / I$-module.

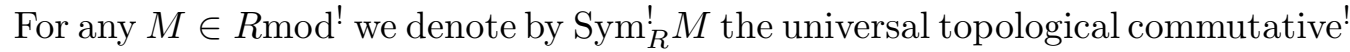
$R$-algebra generated by $M$, i.e., $\operatorname{Sym}_{R}^{!} M=\lim _{R / J} \operatorname{Sym}_{R /}(M / P)$ where the projective limit is taken with respect all pairs $(J, P)$ where $J \subset R$ is an open ideal and $P \subset M$ an open $R$-submodule such that $J \mathcal{L} \subset P$. Our $\operatorname{Sym}_{R}^{!} M$ carries an evident chiral algebra filtration $\left(\operatorname{Sym}_{R}^{!} M\right)_{a}=R \oplus M \oplus \ldots \oplus \operatorname{Sym}_{R}^{! a} M$; here $\operatorname{Sym}_{R}^{! a} M$ is the symmetric power of $M$ in the tensor category $R \bmod !$. The filtration is admissible (due to the universality property of $\operatorname{Sym}_{R}^{!} M$ ), and $\operatorname{gr} \cdot \operatorname{Sym}_{R}^{!} M=\operatorname{Sym}_{R}^{!} M$ is the universal graded topological commutative! $R$-algebra generated by $M$ in degree 1 .

A topological Lie* $R$-algebroid is a topological vector space $\mathcal{L}$ equipped with a Lie $R$-algebroid structure such that the Lie bracket is $\left(\otimes^{*}-\right)$ continuous and the $R$-action on $\mathcal{L}$ is $\otimes$ !-continuous. We demand that $\mathcal{L}$ is a topological Lie* algebra in the sense of 1.4, or, equivalently, that open Lie $R$-subalgebroids of $\mathcal{L}$ form a base of the topology of $\mathcal{L}$. If $\mathcal{L}$ is a topological Lie* $R$-algebroid, then $\operatorname{Sym}_{R}^{!} \mathcal{L}$ is naturally a topological coisson algebra.

Examples. (i) Let $L$ be a topological Lie* algebra that acts continuously on $R$. Then $L_{R}:=R \otimes^{!} L$ is naturally a topological Lie* $R$-algebroid.

(ii) Let $\Omega_{R}:=\varliminf_{R / I} \Omega_{R}$ be the topological $R$-module of continuous differentials 
of $R$. Suppose that $R$ is reasonable, formally smooth, and the topology of $R$ admits a countable base; then $\Omega_{R}$ is a Tate $R$-module (see [D] Th. 6.2(iii)). Let $\Theta_{R}$ be the dual Tate $R$-module. Explicitly, $\Theta_{R}=\lim _{R} \Theta_{R, R / I}$ where for an open ideal $I \subset R$ the topological $R / I$-module $\Theta_{R, R / I}=R / I \underset{R}{\otimes} \Theta_{R}$ consists of all continuous derivations $\theta: R \rightarrow R / I$; the topology of $\Theta_{R, R / I}$ has base formed by $R / I$-submodules that consist of $\theta$ that kill given open ideal $J \subset I$ and finite subset of $R / J$. Our $\Theta_{R}$ is naturally a topological Lie* $R$-algebroid called the tangent algebroid of $R$.

2.3. PBW filtrations. Let $A$ be a topological chiral algebra equipped with a commutative chiral algebra filtration $A$. Set $R:=A_{0}, \mathcal{L}:=\operatorname{gr}_{1} A=A_{1} / A_{0}$. Then $R$ is a topological commutative! algebra and $\mathcal{L}$ is a topological Lie* $R$-algebroid. By the universality property we have an evident morphism of commutative! $R$-algebras, which is automatically a morphism of topological coisson algebras,

$$
\operatorname{Sym}_{R} \mathcal{L} \rightarrow \widehat{\operatorname{gr}} A
$$

called the Poincaré-Birkhoff-Witt map.

Lemma. Suppose that the filtration $A$. is admissible and each map $S y m_{R}^{! n} \mathcal{L} \rightarrow$ $g_{n} A$ is an admissible epimorphism (i.e., an open surjection). Then for every open subspace $P \subset A_{1}$ the closure $\overline{A P}=\overline{A_{\infty} P}$ of the left ideal generated by $P$ is open. Such ideals form a base of the topology of $A$.

Proof. The second assertion is immediate, once we check the first one. By admissibility, it suffices to check that $\overline{A_{n-1} P}$ is open in $A_{n}$ for each $n$. By induction, we know that $\overline{A_{n-1} P} \cap A_{n-1}$ is open in $A_{n-1}$, i.e., we have an open $V \subset A_{n}$ such that $V \cap A_{n-1} \subset \overline{A_{n-1} P} \cap A_{n-1}$. The image $\operatorname{gr}_{n} \overline{A_{n-1} P}$ of $\overline{A_{n-1} P}$ in $\operatorname{gr}_{n} A$ is open: indeed, if an open subspace $T$ of $P$ tends to zero, then $A_{n-1} T$ tends to zero, hence $\operatorname{gr}_{n} \overline{A_{n-1} P}$ contains the image of $\varliminf_{(\lim }\left(\operatorname{gr}_{n-1} A \cdot \operatorname{gr}_{1} P\right) /\left(\operatorname{gr}_{n-1} A \cdot \operatorname{gr}_{1} T\right)$ which is an open subspace in $\operatorname{gr}_{n} A$. Choose $T$ as above such that $A_{n-1} T \subset V$; replacing $V$ by its intersection with the preimage of the open subspace $\operatorname{gr}_{n} \overline{A_{n-1} T}$ of $\mathrm{gr}_{n} A$, we can assume that $V=\left(V \cap A_{n-1}\right)+\left(\overline{A_{n-1} T}\right)$, hence $V \subset \overline{A_{n-1} P}$, q.e.d.

Definition. We say that $A$. satisfies the weak $P B W$ property if $\operatorname{Sym}_{R}^{! n} \mathcal{L} \stackrel{\sim}{\rightarrow} \operatorname{gr}_{n} A$ for each $n$; the strong $P B W$ property means that the filtration is admissible and (2.3.1) is an isomorphism. Such a filtration is referred to as weak, resp. strong, $P B W$ filtration.

Remarks. (i) The strong PBW property asserts the existence of large discrete quotients of $A$. Indeed, it amounts to the following two conditions: (a) The filtration is generated by $A_{1}$, i.e., each $A_{n}, n \geq 1$, equals the closure of $\left(A_{1}\right)^{n}$; (b) Let $P \subset A_{1}$ be an open subspace such that $\operatorname{gr}_{0} P=P \cap R$ is an ideal in $R$ and $\operatorname{gr}_{1} P$ is an $R$-submodule of $\operatorname{gr}_{1} A$. Then one can find an open ideal $I \subset A$ such that $I \cap A_{1} \subset P$ and the projection $\operatorname{gr}_{1} A / \mathrm{gr}_{1} I \rightarrow \mathrm{gr}_{1} A / \mathrm{gr}_{1} P$ lifts to a morphism of algebras gr. $A /$ gr. $I \rightarrow \operatorname{Sym}_{R / \operatorname{gr}_{0} P}\left(\operatorname{gr}_{1} A / \operatorname{gr}_{1} P\right)$.

(ii) I do not know if every admissible weak PBW filtration automatically satisfies the strong PBW property.

2.4. The chiral envelope of a Lie* algebra. The forgetful functor from the category of topological chiral algebras to that of Lie* algebras $\left(A, \mu_{A}\right) \mapsto\left(A,[]_{A}\right)$ 
(see 1.4) admits (as follows easily from 1.5) a left adjoint functor. For a Lie* algebra $L$ we denote by $U^{c h}(L)$ the corresponding chiral enveloping algebra. Explicitly, $U^{c h}(L)$ is the completion of the plain enveloping algebra $U(L)$ with respect to a topology formed by all the left ideals $U(L) P$ where $P \subset L$ is an open vector subspace (it satisfies the conditions of Corollary in 1.4).

Our $U^{c h}(L)$ carries a standard filtration $U^{c h}(L)$. defined as the completion of the standard (Poincaré-Birkhoff-Witt) filtration on $U(L)$. It is admissible and commutative, so the morphism of Lie* algebras $L \rightarrow \operatorname{gr}_{1} U^{c h}(L)$ yields a morphism of topological coisson algebras

$$
\operatorname{Sym}^{!} L \rightarrow \widehat{\operatorname{gr}} U^{c h}(L) .
$$

Lemma. (2.4.1) is an isomorphism, i.e., $U^{\text {ch }}(L)$. is a strong PBW filtration.

Proof. By the usual PBW theorem, for every open Lie subalgebra $P \subset L$ one has gr. $(U(L) / U(L) P)=\operatorname{Sym}^{!} \cdot(L / P)$ (here the quotient $U(L) / U(L) P$ is equipped with the image of the standard filtration). Such $P$ form a base of the topology of $L$ (by the definition of Lie* algebra, see 1.4), and we are done.

2.5. Chiral extensions of a $\mathbf{L i e}^{*}$ algebroid. We want to prove a similar result for a topological Lie* algebroid. First we need to define its enveloping algebra. This requires (just as in the $\mathcal{D}$-module setting of $[\mathrm{BD}] 3.9$ ) an extra structure of chiral extension that we are going to define.

So let $R$ be a topological commutative! algebra, $\mathcal{L}$ a topological Lie* $R$-algebroid.

Consider for a moment $R$ as a commutative algebra and $\mathcal{L}$ as a Lie $R$-algebroid in the tensor category $\mathcal{T o p}^{*}$. Let $\mathcal{L}^{b}$ be a Lie $R$-algebroid extension of $\mathcal{L}$ by $R$ in $\mathcal{T}_{\text {op }}{ }^{*}$; below we call such $\mathcal{L}^{b}$ simply a topological $R$-extension of $\mathcal{L}$. Explicitly, our $\mathcal{L}^{b}$ is an extension of topological vector spaces

$$
0 \rightarrow R \stackrel{i}{\rightarrow} \mathcal{L}^{b} \stackrel{\pi}{\rightarrow} \mathcal{L} \rightarrow 0
$$

together with a Lie $R$-algebroid structure on $\mathcal{L}^{b}$ such that $\pi$ is a morphism of Lie $R$-algebroids, $i$ is a morphism of $R$-modules, $1^{b}:=i(1)$ is a central element of $\mathcal{L}^{b}$; we also demand that $\mathcal{L}^{b}$ is a Lie* algebra and the $R$-action on $\mathcal{L}^{b}$ is $\left(\otimes^{*}\right.$-) continuous.

Exercise. $\mathcal{L}^{b}$ is automatically a topological Lie* algebra (see 1.4).

Our $\mathcal{L}^{b}$ is automatically an $R$-bimodule where the right $R$-action is defined by formula $\ell^{b} r=r \ell^{b}+\ell(r)$, where $\ell^{b} \in \mathcal{L}^{b}, r \in R, \ell:=\pi\left(\ell^{b}\right)$, and $\ell(r) \in R \subset \mathcal{L}^{b}$; the right $R$-action is continuous as well.

Definition. (a) $\mathcal{L}^{b}$ is called a classical $R$-extension of $\mathcal{L}$ if $\mathcal{L}^{b}$ is a topological $R$ algebroid, i.e., the (left) $R$-action on $\mathcal{L}$ is $\otimes^{!}$-continuous, so we have $R \otimes ! \mathcal{L}^{b} \rightarrow \mathcal{L}^{b}$. (b) $\mathcal{L}^{b}$ is called a chiral $R$-extension of $\mathcal{L}$ if the left and right $R$-actions on $\mathcal{L}^{b}$ are $\vec{\otimes}$-continuous, i.e., we have $R \vec{\otimes} \mathcal{L}^{b} \rightarrow \mathcal{L}^{b}$ and $\mathcal{L}^{b} \vec{\otimes} R \rightarrow \mathcal{L}^{b}$.

Example. As in 2.3, let $A$ be a chiral algebra equipped with a commutative filtration $A$., so $R:=A_{0}$ is a commutative! algebra and $\mathcal{L}:=\operatorname{gr}_{1} A$ is $\operatorname{Lie}^{*} A_{0^{-}}$ algebroid. Set $\mathcal{L}^{b}:=A_{1}$; this is an $R$-extension of $\mathcal{L}$. The Lie bracket on $A_{1}$, the 
left $A_{0}$-action on $A_{1}$, and the adjoint action of $A_{1}$ on $A_{0}$ make $\mathcal{L}^{b}$ a topological $R$ extension of the Lie* $R$-algebroid $\mathcal{L}$. Since the right $R$-action on $\mathcal{L}^{b}$ equals the right $A_{0}$-action on $A_{1}$ that comes from the algebra structure on $A$, it is $\vec{\otimes}$-continuous. Therefore $\mathcal{L}^{b}$ is a chiral $R$-extension of $\mathcal{L}$.

The topological $R$-extensions of $\mathcal{L}$ form naturally a Picard groupoid $\mathcal{P}(\mathcal{L})$; the operation is the Baer sum. More precisely, $\mathcal{P}(\mathcal{L})$ is a $k$-vector space in groupoids: For $\mathcal{L}^{b_{1}}, \mathcal{L}^{b_{2}} \in \mathcal{P}(\mathcal{L})$ and $a_{1}, a_{2} \in k$ the topological $R$-extension $\mathcal{L}^{a_{1} b_{1}+a_{2} b_{2}}$ is defined as the the push-out of $0 \rightarrow R \times R \rightarrow \mathcal{L}^{b_{1}} \times{ }_{\mathcal{L}} \mathcal{L}^{b_{2}} \rightarrow \mathcal{L} \rightarrow 0$ by the map $R \times R \rightarrow R$, $\left(r_{1}, r_{2}\right) \mapsto a_{1} r_{1}+a_{2} r_{2}$; the Lie $R$-algebroid structure on it is defined by the condition that the canonical map $\mathcal{L}^{b_{1}} \times{ }_{\mathcal{L}} \mathcal{L}^{b_{2}} \rightarrow \mathcal{L}^{a_{1} b_{1}+a_{2} b_{2}}$ is a morphism of Lie $R$-algebroids. Let $\mathcal{P}^{c l}(\mathcal{L}), \mathcal{P}^{c h}(\mathcal{L}) \subset \mathcal{P}(\mathcal{L})$ be the subgroupoids of classical and chiral $R$-extensions.

Lemma. $\mathcal{P}^{c l}(\mathcal{L})$ is a Picard subgroupoid (actually, a k-vector subspace) of $\mathcal{P}(\mathcal{L})$. If $\mathcal{P}^{c h}(\mathcal{L})$ is non-empty, then it is a $\mathcal{P}^{c l}(\mathcal{L})$-torsor.

Proof. Let $\mathcal{L}^{b}$ be a topological $R$-extension of $\mathcal{L}$; fix $\lambda \in k$. For $\ell^{b} \in \mathcal{L}^{b}, r \in R$ set $\ell^{b} \cdot{ }_{\lambda} r=r \ell^{b}+\lambda \ell(r)$. The operation $\cdot_{\lambda}$ is a right $R$-module structure on $\mathcal{L}^{b}$ (which commutes with the left $R$-module structure). Notice that $\cdot_{1}$ is the old right $R$-action on $\mathcal{L}^{b}$, and $\cdot 0$ is the left $R$-action.

Suppose we have $\mathcal{L}^{b_{i}} \in \mathcal{P}(\mathcal{L})$ and $\lambda_{i}, a_{i} \in k$; here $i=1,2$. The right $R$-actions $\cdot \lambda_{i}$ on $\mathcal{L}^{b_{i}}$ yield a right $R$-action on $\mathcal{L}^{b_{1}} \times{ }_{\mathcal{L}} \mathcal{L}^{b_{2}}$, hence a right $R$-action on $\mathcal{L}^{a_{1} b_{1}+a_{2} b_{2}}$ such that the canonical map $\mathcal{L}^{b_{1}} \times{ }_{\mathcal{L}} \mathcal{L}^{b_{2}} \rightarrow \mathcal{L}^{a_{1} b_{1}+a_{2} b_{2}}$ is a morphism of right $R$ modules. The latter right $R$-action on $\mathcal{L}^{a_{1} b_{1}+a_{2} b_{2}}$ clearly equals $\cdot a_{1} \lambda_{1}+a_{2} \lambda_{2}$.

If the right $R$-actions $\cdot_{\lambda_{i}}$ on $\mathcal{L}^{b_{i}}$ are $\vec{\otimes}$-continuous, then the right $R$-actions on $\mathcal{L}^{b_{1}} \times{ }_{\mathcal{L}} \mathcal{L}^{b_{2}}$ and $\mathcal{L}^{a_{1} b_{1}+a_{2} b_{2}}$ are $\vec{\otimes}$-continuous as well. Therefore the right $R$-action $\cdot a_{1} \lambda_{1}+a_{2} \lambda_{2}$ on $\mathcal{L}^{a_{1} b_{1}+a_{2} b_{2}}$ is $\vec{\otimes}$-continuous.

Let us call $\mathcal{L}^{b}$ a $\lambda$-chiral $R$-extension if the left $R$-action $R \otimes \mathcal{L}^{b} \rightarrow \mathcal{L}^{b}$ and the right action $\cdot_{\lambda}: \mathcal{L}^{b} \otimes R \rightarrow \mathcal{L}^{b}$ are $\vec{\otimes}$-continuous. E.g., 0-chiral extension is the same as classical extension, and 1-chiral extension is the same as chiral extension. We have checked that if $\mathcal{L}^{b_{i}}$ are $\lambda_{i}$-chiral $R$-extensions, then $\mathcal{L}^{a_{2} b_{1}+a_{2} b_{2}}$ is an $a_{1} \lambda_{1}+a_{2} \lambda_{2^{-}}$ chiral extension. In particular: If $\mathcal{L}^{b_{i}}$ are classical extensions, then $\mathcal{L}^{a_{1} b_{1}+a_{2} b_{2}}$ is a classical extension for every $a_{i} \in k$. If $\mathcal{L}^{b_{1}}$ is a classical extension, $\mathcal{L}^{b_{2}}$ is a chiral one, then $\mathcal{L}^{b_{1}+b_{2}}$ is a chiral extension. If $\mathcal{L}^{b_{i}}$ are chiral extensions, then $\mathcal{L}^{b_{1}-b_{2}}$ is a classical extension. We are done.

Exercises. (i) A classical extension $\mathcal{L}^{b}$ of $\mathcal{L}$ is a chiral extension if and only if for every open ideal $I \subset R$ there is an open ideal $J \subset R$ such that $\mathcal{L}(J) \subset I$. (ii) For a topological $R$-extension $\mathcal{L}^{b}$ let $\mathcal{L}^{b \tau}$ be the "inverse" $R$-extension: so we have an identification of topological vector spaces $\mathcal{L}^{b \tau} \stackrel{\sim}{\longrightarrow} \mathcal{L}^{b}$ which commutes with $i$ 's and anticommutes with $\pi$ 's in (2.5.1), interchanges the left and right $R$-module structures, and identifies the Lie bracket with on $\mathcal{L}^{b \tau}$ with minus Lie bracket on $\mathcal{L}^{b}$. Show that if $\mathcal{L}^{b}$ is a classical extension, then $\mathcal{L}^{b \tau}$ is a chiral extension if and only if for every open ideal $I \subset R$ there is an open $P \subset \mathcal{L}$ such that $P(R) \subset I$.

2.6. The enveloping algebra of a chiral Lie algebroid. Let $\mathcal{L}^{b}$ be a chiral extension of a Lie* $R$-algebroid $R$; we call a triple $\left(R, \mathcal{L}, \mathcal{L}^{b}\right)$ a topological chiral Lie algebroid. These objects form naturally a category $\mathcal{C} \mathcal{L}(\mathcal{T}$ op $)$. 
Let $\mathcal{C} \mathcal{A}^{f c}(\mathcal{T} o p)$ be the category of topological chiral algebras equipped with a commutative filtration. By Example in 2.5, we have a functor $\mathcal{C} \mathcal{A}^{\text {fc }}(\mathcal{T}$ op $) \rightarrow$ $\mathcal{C} \mathcal{L}\left(\mathcal{T}_{\text {op }}\right),\left(A, A_{\text {. }}\right) \mapsto\left(A_{0}, \operatorname{gr}_{1} A, A_{1}\right)$.

Proposition. This functor admits a left adjoint $\mathcal{C} \mathcal{L}(\mathcal{T}$ op $) \rightarrow \mathcal{C} \mathcal{A}^{\text {fc }}\left(\mathcal{T}_{\text {op }}\right)$.

We denote this adjoint functor by $\left(R, \mathcal{L}, \mathcal{L}^{b}\right) \mapsto U_{R}^{c h}(\mathcal{L})^{b}$ and call $U_{R}^{c h}(\mathcal{L})^{b}$ the chiral enveloping algebra of $\mathcal{L}^{b}$. The commutative filtration $U_{R}^{c h}(\mathcal{L})^{b}$. on $U_{R}^{c h}(\mathcal{L})^{b}$ is referred to as the standard filtration.

Proof. Let us define $U_{R}^{c h}(\mathcal{L})^{b}$ as a universal chiral algebra equipped with a continuous map $\varphi^{b}: \mathcal{L}^{b} \rightarrow U_{R}^{c h}(\mathcal{L})^{b}$ such that $\varphi^{b}$ is a morphism of Lie algebras, its restriction to $R \subset \mathcal{L}^{b}$ is a morphism of chiral (or associative) algebras, and $\varphi^{b}$ is a morphism of $R$-bimodules ${ }^{6}$ (with respect to $\left.\varphi^{b}\right|_{R}$ ). To construct it explicitly, consider the "abstract" enveloping algebra $U_{R}(\mathcal{L})^{b}$ of $\mathcal{L}^{b}$, i.e., take copies of $R, \mathcal{L}$, and $\mathcal{L}^{b}$ equipped with discrete topologies; then $U_{R}(\mathcal{L})^{b}$ is the corresponding chiral enveloping algebra (its topology is discrete). Now $U_{R}(\mathcal{L})^{b}$ carries a linear topology whose base is formed by all left ideals $U_{R}(\mathcal{L})^{b}(P+I)$ where $P \subset L$ and $I \subset R$ are open subspaces. Shrinking $P, I$ if necessary, one can assume that $P$ is an open Lie subalgebra of $\mathcal{L}^{b}$ and $I$ an open $P$-stable ideal of $R$. This topology satisfies the conditions of Corollary from 1.4, so the corresponding completion is a chiral algebra which equals $U_{R}^{c h}(\mathcal{L})^{b}$ due to the universality property.

The standard filtration is defined in the usual manner: $U_{R}^{c h}(\mathcal{L})_{n}^{b}$ is the closure of the image of $R$ for $n=0$, and is the closure of the image of $n$th power of the image of $\mathcal{L}^{b}$ if $n \geq 1$. It is clearly admissible and commutative. As an object of $\mathcal{C} \mathcal{A}^{f c}(\mathcal{T} o p)$, our $U_{R}^{c h}(\mathcal{L})^{b}$ evidently satisfies the universality property of the statement, and we are done.

Remark. The above explicit construction of $U_{R}^{c h}(\mathcal{L})^{b}$ implies that a discrete $U_{R}^{c h}(\mathcal{L})^{b}$-module is the same as a vector space $M$ equipped with a continuous Lie algebra action of $\mathcal{L}^{b}$ such that the action of $R \subset \mathcal{L}^{b}$ is a unital $R$-module structure on $M$, and for $r \in R, \ell^{b} \in \mathcal{L}^{b}, m \in M$ one has $\left(r \ell^{b}\right) m=r\left(\ell^{b} m\right)$.

2.7. Rigidified chiral extensions. Let $R$ be a topological commutative! algebra, $L$ a topological Lie* algebra that acts on $R$ in a continuous way. Then $L_{R}:=R \otimes ! L$ is naturally a topological Lie* $R$-algebroid.

Lemma. There is a unique, up to a unique isomorphism, chiral extension $L_{R}^{b}$ equipped with a Lie* algebra morphism $L \rightarrow L_{R}^{b}$ which lifts the embedding $L \hookrightarrow L_{R}$.

Proof. We construct $\mathcal{L}^{b}$ explicitly; the uniqueness is clear from the construction.

Forgetting for a moment about the topologies, consider the $L$-rigidified Lie $R$ algebroid $L_{R}^{\delta}=R \otimes L$ and its trivialized $R$-extension $L_{R}^{\sharp}=L_{R}^{\delta} \oplus R$. Our $L_{R}^{\sharp}$ contains $L$ as a Lie subalgebra; as in 2.5, it is naturally an $R$-bimodule. For open subspaces $P \subset L$ and $I \subset R$ let $\langle P, I\rangle \subset L_{R}^{\sharp}$ be the vector subspace formed by linear combinations of all vectors $r p, \ell i, i^{\prime} \in L_{R}^{\sharp}$ where $p \in P, i, i^{\prime} \in I$ and $\ell, r$ are

\footnotetext{
${ }^{6}$ It suffices to demand that $\varphi^{b}$ is a morphism of either left or right $R$-modules.
} 
arbitrary elements of $L, R$. These subspaces form a topology on $L_{R}^{\sharp}$; we define $L_{R}^{b}$ as the corresponding completion.

Notice that the subspaces $\langle P, I\rangle$ with $P \subset L$ an open Lie subalgebra and $I \subset R$ an open ideal preserved by the $P$-action form a base of the topology on $L_{R}^{\sharp}$. For such $P, I$ one has $\langle P, I\rangle=R P+L_{R}^{\sharp} I$. One also has $\langle P, I\rangle \cap R=I$, so $L_{R}^{b}$ is an extension of $R \otimes ! L$ by $R$. The evident morphism $L \rightarrow L_{R}^{b}$ is continuous.

For $P, I$ as above the subspace $\langle P, I\rangle$ is a Lie subalgebra and a left $R$-submodule (i.e., a Lie $R$-subalgebroid) of $L_{R}^{\sharp}$. The Lie bracket and the left and right actions of $R$ on $L_{R}^{\sharp}$ are continuous with respect to our topology, hence $L_{R}^{b}$ is a topological $R$-extension of $L_{R}$ in the sense of 2.5. Clearly it is a chiral $R$-extension, q.e.d.

Remark. Here is another description of $L_{R}^{b}$ as a mere topological extension. The map $L \otimes R \oplus R \otimes L \rightarrow L_{R}^{\sharp}, \ell \otimes r+r^{\prime} \otimes \ell^{\prime} \mapsto \ell r+r^{\prime} \ell^{\prime}$, extends by continuity to a continuous morphism $L \vec{\otimes} R \oplus R \vec{\otimes} L \rightarrow L_{R}^{b}$. It identifies $L_{R}^{b}$ with the quotient of $L \vec{\otimes} R \oplus R \vec{\otimes} L \oplus R$ modulo the closed subspace generated by vectors $\ell \otimes r-r \otimes \ell-\ell(r)$. Equivalently, consider the extension $0 \rightarrow L \otimes^{*} R \stackrel{(+,-)}{\longrightarrow} L \vec{\otimes} R \oplus R \vec{\otimes} L \stackrel{(+,+)}{\longrightarrow} R \otimes !$ (see (1.1.2)); then $0 \rightarrow R \rightarrow L_{R}^{b} \rightarrow L_{R} \rightarrow 0$ is its its push-out by the $L$-action map $L \otimes^{*} R \rightarrow R$.

2.8. The de Rham-Chevalley chiral Lie* algebroid. Let $R$ be a reasonable topological algebra, $\mathcal{L}$ a Tate $R$-module, $\mathcal{L}^{*}$ the dual Tate $R$-module (see [D]).

Exercise. The $R$-module of all continuous $R$ - $n$-linear maps $\mathcal{L} \times \ldots \times \mathcal{L} \rightarrow R$ identifies naturally with the $n$th tensor power of $\mathcal{L}^{*}$ in $R \bmod$ !.

Suppose we have a Lie* $R$-algebroid structure on $\mathcal{L}$. Forget for a moment about the topologies and consider $\mathcal{L}$ as a mere Lie $R$-algebroid. We have the corresponding de Rham-Chevalley complex $\mathcal{C}_{R}(\mathcal{L})$ : this is a commutative DGA whose $n$th term equals $\operatorname{Hom}_{R}\left(\Lambda_{R}^{n} \mathcal{L}, R\right)$ and the differential is given by the usual formula. By the exercise, the continuous maps form a graded subalgebra $\mathcal{C}=\mathcal{C}_{R}(\mathcal{L})$ of $\mathcal{C}_{R}(\mathcal{L})$ which equals $\operatorname{Sym}_{R}^{!}\left(\mathcal{L}^{*}[-1]\right)$.

Lemma. The de Rham-Chevalley differential preserves the subalgebra $\mathcal{C}$ and is continuous on it, so $\mathcal{C}$ is a topological commutative DG algebra.

Exercise. A Lie* $R$-algebroid structure on $\mathcal{L}$ amounts to a differential on the topological graded algebra $\operatorname{Sym}_{R}^{!}\left(\mathcal{L}^{*}[-1]\right)$.

Our $\mathcal{C}$ carries a natural topological DG Lie* $\mathcal{C}$-algebroid $\mathcal{L}_{\mathcal{C}}$ (cf. [BD] 3.9.16). To construct it, consider $\mathcal{L}$ as a mere Lie* algebra. It acts on $\mathcal{C}$ by transport of structure, and this action extends naturally to an action of the contractible Lie* DG algebra $\mathcal{L}_{\dagger}:=\mathcal{C}$ one $($ id $: \mathcal{L} \rightarrow \mathcal{L})$ : namely, the component $\mathcal{L}[1]$ acts by the evident $R$-linear derivations of $\operatorname{Sym}_{R}^{!}\left(\mathcal{L}^{*}[-1]\right)$. Thus we have the corresponding DG Lie* $\mathcal{C}$-algebroid $\mathcal{L}_{\dagger \mathcal{C}}$. By construction, $\mathcal{L}_{\dagger \mathcal{C}}^{<-1}=0$ and $\mathcal{L}_{\dagger \mathcal{C}}^{-1}=R \otimes ! \mathcal{L}$. Let $\mathcal{K}$ be the closed DG $\mathcal{C}$-submodule of $\mathcal{L}_{\dagger \mathcal{C}}$ generated by $\mathcal{K}^{-1} \subset \mathcal{L}_{\dagger \mathcal{C}}^{-1}$ defined as the kernel of the product map $R \otimes ! \mathcal{L} \rightarrow \mathcal{L}$. Since $\mathcal{K}^{-1}$ acts trivially on $\mathcal{C}$ and is normalized by the adjoint action of $\mathcal{L}_{\dagger}$, we see that $\mathcal{K}$ is a DG ideal in the Lie* $\mathcal{C}$-algebroid $\mathcal{L}_{\dagger \mathcal{C}}$. The promised $\mathcal{L}_{\mathcal{C}}$ is the quotient $\mathcal{L}_{\dagger \mathcal{C}} / \mathcal{K}$. 
Set $\mathcal{L}_{\dagger \mathcal{C}+}:=\operatorname{Sym}^{!} \cdot\left(\mathcal{L}^{*}[-1]\right) \otimes_{R} \mathcal{L}[1]$; we consider it as the graded Lie* $\mathcal{C}$-algebroid generated by the action of the Lie* subalgebra $\mathcal{L}[1]$ of $\mathcal{L}_{\dagger}$ on $\mathcal{C}$. The embedding into $\mathcal{L}_{\dagger \mathcal{C}}$ identifies it with $\mathcal{C} \cdot \mathcal{L}_{\dagger \mathcal{C}}^{-1}$, which is a graded submodule of $\mathcal{L}_{\dagger \mathcal{C}}$ not preserved by the differential: in fact, the Kodaira-Spencer map $\mathcal{L}_{\dagger \mathcal{C}+} \rightarrow \mathcal{L}_{\dagger \mathcal{C}} / \mathcal{L}_{\dagger \mathcal{C}+}$, $\ell \mapsto d(\ell) \bmod \mathcal{L}_{\mathcal{C}_{+}}$, is an isomorphism. Similarly, consider $\mathcal{K}_{+}:=\mathcal{K} \cap \mathcal{L}_{\dagger \mathcal{C}+}$; then $\mathcal{K}_{+}=\operatorname{Sym}^{!} \cdot\left(\mathcal{L}^{*}[-1]\right) \otimes_{R}^{!} \mathcal{K}^{-1}$ and the Kodaira-Spencer map $\mathcal{K}_{+} \rightarrow \mathcal{K} / \mathcal{K}_{+}$is an isomorphism. Therefore $\mathcal{L}_{\mathcal{C}+}:=$ the closed $\mathcal{C}$-submodule of $\mathcal{L}_{\mathcal{C}}$ generated by $\mathcal{L}_{\mathcal{C}}^{-1}$, equals $\operatorname{Sym}_{R}^{!}\left(\mathcal{L}^{*}[-1]\right) \otimes_{R}^{!} \mathcal{L}_{\mathcal{C}+}^{-1}=\operatorname{Sym}_{R}^{!}\left(\mathcal{L}^{*}[-1]\right) \otimes_{R}^{!} \mathcal{L}[1]$, and the Kodaira-Spencer map $\mathcal{L}_{\mathcal{C}+} \rightarrow \mathcal{L}_{\mathcal{C}} / \mathcal{L}_{\mathcal{C}+}$ is an isomorphism.

Remark. We see that the Lie* $R$-algebroid $\mathcal{L}_{\mathcal{C}}^{0}$ is an extension of $\mathcal{L}$ by $\mathcal{L}_{\mathcal{C}+}^{0}=$ $\mathcal{L}^{*} \otimes_{R}^{!} \mathcal{L}$. It acts naturally on the Tate $R$-module $\mathcal{L}_{\mathcal{C}}^{-1}=\mathcal{L}$ by the adjoint action. The restriction of this action to the Lie* $R$-algebra $\mathcal{L}^{*} \otimes_{R}^{!} \mathcal{L}$ identifies $\mathcal{L}^{*} \otimes_{R} \mathcal{L}$ with Lie algebra $\mathfrak{g l}_{R}(\mathcal{L})$ of continuous $R$-linear endomorphisms of $\mathcal{L}$ as in 1.2.

Proposition. The topological DG Lie* $\mathcal{C}$-algebroid $\mathcal{L}_{\mathcal{C}}$ admits a unique topological chiral $D G$ extension $\mathcal{L}_{\mathcal{C}}^{b}$.

Proof (cf. [BD] 3.9.17). Existence. Consider the rigidified DG chiral extension $\mathcal{L}_{\dagger \mathcal{C}}^{b}$ of the Lie* $\mathcal{C}$-algebroid $\mathcal{L}_{\dagger \mathcal{C}}$. Let $\tilde{\mathcal{K}} \subset \mathcal{L}_{\dagger \mathcal{C}}^{b}$ be the closed DG left $\mathcal{C}$ submodule of $\mathcal{L}_{\dagger \mathcal{C}}^{b}$ generated by $\tilde{\mathcal{K}}^{-1}:=$ the preimage of $\mathcal{K}^{-1}$ by the projection $\mathcal{L}_{\dagger \mathcal{C}}^{b-1} \rightarrow \mathcal{L}_{\dagger \mathcal{C}}^{-1}$. (which is an isomorphism since $\mathcal{C}^{-1}=0$ ). Then $\tilde{\mathcal{K}}$ is preserved by the adjoint action of $\mathcal{L}_{\dagger}$ (since $\tilde{\mathcal{K}}^{-1}$ is) and $\tilde{\mathcal{K}}$ acts trivially on $\mathcal{C}$, hence $\tilde{\mathcal{K}}$ is preserved by the adjoint action of $\mathcal{L}_{\dagger \mathcal{C}}^{b}$.

We will show in a moment that $\tilde{\mathcal{K}} \cap \mathcal{C}=0$. Then $\mathcal{L}_{\mathcal{C}}^{b}:=\mathcal{L}_{\dagger \mathcal{C}}^{b} / \tilde{\mathcal{K}}$ is a chiral DG extension of $\mathcal{L}_{\mathcal{C}}$, and we are done.

Our $\tilde{\mathcal{K}} \cap \mathcal{C}$ is a DG ideal of $\mathcal{C}$. It is closed with respect to the action of $\mathcal{L}[1] \subset \mathcal{L}_{\dagger}$, so it suffices to check that $\tilde{\mathcal{K}}^{0} \cap R=0$. Notice that $\tilde{\mathcal{K}}^{0}$ is sum of the closures of $\mathcal{C}^{1} \cdot \tilde{\mathcal{K}}^{-1}$ and $d\left(\tilde{\mathcal{K}}^{-1}\right)$. The composition $\tilde{\mathcal{K}}^{-1} \stackrel{d}{\rightarrow} \tilde{\mathcal{K}}^{0} \rightarrow \mathcal{K}^{0} / \mathcal{K}_{+}^{0}$ is a closed embedding, so $d\left(\tilde{\mathcal{K}}^{-1}\right)$ is a closed subspace of $\mathcal{L}_{\dagger \mathcal{C}}^{b 0}$ which does not intersect $\mathcal{L}_{\dagger \mathcal{C}+}^{b 0}$. It remains to prove that the closure of $\mathcal{C}^{1} \cdot \tilde{\mathcal{K}}^{-1} \subset \mathcal{L}_{\uparrow \mathcal{C}+}^{b 0}$ does not intersect $R$. The map $\mathcal{C}^{1} \vec{\otimes} \tilde{\mathcal{K}}^{-1} \oplus \tilde{\mathcal{K}}^{-1} \vec{\otimes} \mathcal{C}^{1} \rightarrow \mathcal{L}_{\dagger \mathcal{C}+}^{b 0}, c \otimes k+k^{\prime} \otimes c^{\prime} \mapsto c k+k^{\prime} c^{\prime}$, vanishes on the subspace $\mathcal{C}^{1} \otimes^{*} \tilde{\mathcal{K}}^{-1}$ (see (1.1.2)) since $\mathcal{K}^{-1}$ acts trivially on $\mathcal{C}$. So, by (1.1.2), the product map $\mathcal{C}^{1} \vec{\otimes} \tilde{\mathcal{K}}^{-1} \rightarrow \mathcal{L}_{\dagger \mathcal{C}+}^{b 0}$ extends by continuity to $\mathcal{C}^{1} \otimes ! \tilde{\mathcal{K}}^{-1} \rightarrow \mathcal{L}_{\dagger \mathcal{C}+}^{b 0}$ and, by $R$ bilinearity, to $\mu: \mathcal{C}^{1} \otimes_{R}^{!} \tilde{\mathcal{K}}^{-1} \rightarrow \mathcal{L}_{\dagger \mathcal{C}+}^{b 0}$. We know that the composition $\mathcal{C}^{1} \otimes_{R}^{!} \mathcal{K}^{-1}=$ $\mathcal{C}^{1} \otimes_{R}^{!} \tilde{\mathcal{K}}^{-1} \stackrel{\mu}{\rightarrow} \mathcal{L}_{\dagger \mathcal{C}+}^{b 0} \rightarrow \mathcal{L}_{\dagger \mathcal{C}+}^{0}$ is a closed embedding, so $\mu$ is a closed embedding whose image does not intersect $R$, q.e.d.

Uniqueness. By the proposition in 2.5, it suffices to show that every classical DG extension $\mathcal{L}_{\mathcal{C}}^{c l}$ of $\mathcal{L}_{\mathcal{C}}$ admits a unique splitting. The uniqueness of the splitting is clear since $\mathcal{L}_{\mathcal{C}}^{\text {cl-1 }} \stackrel{\sim}{\rightarrow} \mathcal{L}_{\mathcal{C}}^{-1}$ and $\mathcal{L}_{\mathcal{C}}$ is generated by $\mathcal{L}_{\mathcal{C}}^{-1}$ as a DG Lie* $\mathcal{C}_{\text {-algebroid. }}$ To construct one, consider the embedding $\mathcal{L}[1] \hookrightarrow \mathcal{L}_{\mathcal{C}}^{-1}=\mathcal{L}_{\mathcal{C}}^{c l-1}$. It extends to a morphism of complexes $\mathcal{L}_{\dagger}=\mathcal{C}$ one $\left(\operatorname{id}_{\mathcal{L}}\right) \rightarrow \mathcal{L}_{\mathcal{C}}^{c l}$ which is a morphism of Lie* algebras (as an immediate computation shows). By universality, we get a morphism of DG Lie* $^{*} \mathcal{C}$-algebroids $\mathcal{L}_{\dagger \mathcal{C}} \rightarrow \mathcal{L}_{\mathcal{C}}^{c l}$ which vanishes on $\mathcal{K}^{-1}$. Thus it vanishes on $\mathcal{K}$, hence we get a promised splitting $\mathcal{L}_{\mathcal{C}} \rightarrow \mathcal{L}_{\mathcal{C}}^{c l}$. 
Remark. By the $R$-extension property, the Lie* bracket between $\mathcal{L}_{\mathcal{C}}^{b-1}$ and $\mathcal{C}^{1} \subset$ $\mathcal{L}_{\mathcal{C}}^{b 1}$ equals the standard pairing $\mathcal{L} \times \mathcal{L}^{*} \rightarrow R$. Therefore the subalgebra of $U_{\mathcal{C}}^{c h}\left(\mathcal{L}_{\mathcal{C}}\right)^{b}$ generated by these submodules equals the Clifford algebra $\mathrm{Cl}=\mathrm{Cl}_{R}\left(\mathcal{L}^{*} \oplus \mathcal{L}\right)$ of the Tate $R$-module $\mathcal{L}^{*} \oplus \mathcal{L}$ equipped with the usual hyperbolic quadratic form. It contains the Lie* $R$-subalgebra $\mathcal{L}_{\mathcal{C}}^{b 0}$ which is the Clifford $R$-extension $\mathfrak{g l}_{R}(\mathcal{L})^{C l}$ of $\mathfrak{g l}_{R}(\mathcal{L})$.

2.9. D-modules. The most interesting special case of the construction from 2.8 is that of $\mathcal{L}=\Theta_{R}$ for $R$ as in Example (ii) in 2.2. Then $\mathcal{C}$ is the de Rham DG algebra of $R$.

Remark. In the vertex (or chiral) algebra setting the enveloping algebra $U_{\mathcal{C}}^{c h}\left(\Theta_{R}\right)^{b}$ was first considered in [MSV] under the name of the chiral de Rham complex; for other, essentially equivalent, constructions (in slightly different settings) see [BD] and $[\mathrm{KV}]$.

Consider for a moment $U_{\mathcal{C}}^{c h}\left(\Theta_{R}\right)^{b}$ as a non-graded topological chiral algebra (which requires an evident completion). The category of discrete $U_{\mathcal{C}}^{c h}\left(\Theta_{R}\right)^{b}$-modules plays the role of the category of $\mathcal{D}$-modules on the ind-scheme $\operatorname{Spec} R$ (this construction, mentioned in $[\mathrm{D}] 6.3 .9$, generalizes the constructions from $[\mathrm{D}]$ and $[\mathrm{KV}]$ ).

Exercises. (i) Suppose that $R$ is discrete. Show that for a discrete $U_{\mathcal{C}}^{c h}\left(\Theta_{R}\right)^{b}-$ module $M$ the subspace $M^{\ell} \subset M$ of elements killed by $\Theta_{R}[1]=\mathcal{L}_{\mathcal{C}}^{b-1}$ is naturally a left $\mathcal{D}$-module on $\operatorname{Spec} R$, i.e., an $R$-module equipped with a flat connection. The functor $M \mapsto M^{\ell}$ is an equivalence between the category of discrete $U_{\mathcal{C}}^{c h}\left(\Theta_{R}\right)^{b}$ modules and that of left $\mathcal{D}$-modules.

(ii) Suppose that $R$ is projective limit of $k$-algebras of finite type, so one has a natural notion of right $\mathcal{D}$-module. Show that for a discrete $U_{\mathcal{C}}^{c h}\left(\Theta_{R}\right)^{b}$-module $M$ the subspace $M^{r} \subset M$ of elements killed by $\mathcal{C} \geq 1$ is naturally a right $\mathcal{D}$-module, and the functor $M \mapsto M^{r}$ is an equivalence between the category of discrete $U_{\mathcal{C}}^{c h}\left(\Theta_{R}\right)^{b}-$ modules and that of right $\mathcal{D}$-modules.

More generally, suppose $R$ is arbitrary, and we have a fermion module $V$ in the sense of [D] 5.4.1 over the Clifford algebra $\mathrm{Cl}$ from the second remark in 2.8. Then $V$ yields a chiral extension $\mathcal{L}^{V}$ of $\mathcal{L}$ defined as follows (cf. [BD] 3.9.20). The Lie* subalgebra $\mathcal{L}_{\mathcal{C}}^{b 0}$ normalizes $\mathrm{Cl} \subset U_{\mathcal{C}}^{c h}\left(\mathcal{L}_{\mathcal{C}}\right)^{b}$, and its adjoint action on $\mathrm{Cl}$ factors through $\mathcal{L}_{\mathcal{C}}^{0}{ }^{7}$ Let $\mathcal{L}_{\mathcal{C}}^{0 V}$ be the set of pairs $\left(\tau, \tau_{V}\right)$ where $\tau \in \mathcal{L}_{\mathcal{C}}^{0}$ and $\tau_{V}$ is a lifting of $\tau$ to $V$, i.e., a continuous endomorphism of $V$ such that $\tau_{V}(c v)=\tau(c) v+c \tau_{V}(v)$ for $c \in \mathrm{Cl}, v \in V$. One shows easily that $\mathcal{L}_{\mathcal{C}}^{0 V}$ is naturally a Lie* $R$-algebroid which is an $R$-extension of $\mathcal{L}_{\mathcal{C}}^{0}$. Notice that the action on $V$ of $\mathfrak{g l}_{R}(\mathcal{L})^{C l} \subset \mathrm{Cl}$ identifies the restriction of $\mathcal{L}_{\mathcal{C}}^{0 V}$ to $\mathfrak{g l}_{R}(\mathcal{L})$ with $\mathfrak{g l}_{R}(\mathcal{L})^{C l}$. Let $\mathcal{L}_{\mathcal{C}}^{0 b V}$ be the Baer difference of the $R$-extensions $\mathcal{L}_{\mathcal{C}}^{0 b}$ and $\mathcal{L}_{\mathcal{C}}^{0 V}$; we see that it splits naturally over $\mathfrak{g l}_{R}(\mathcal{L}) \subset \mathcal{L}_{\mathcal{C}}^{0}$, so we have a chiral Lie* $R$-extension $\mathcal{L}^{V}:=\mathcal{L}_{\mathcal{C}}^{0 b V} / \mathfrak{g l}_{R}(\mathcal{L})$ of $\mathcal{L}$.

Exercise. For a discrete $U_{\mathcal{C}}^{c h}\left(\Theta_{R}\right)^{b}$-module $M$ the natural action of $\mathcal{L}_{\mathcal{C}}^{0 b V}$ on $M^{V}:=\operatorname{Hom}_{\mathrm{Cl}}(V, M)$ factors through $\mathcal{L}^{V}$, and the functor $M \mapsto M^{V}$ is an equivalence between the category of discrete $U_{\mathcal{C}}^{c h}\left(\Theta_{R}\right)^{b}$-modules and that of discrete modules over the enveloping chiral algebra $U_{R}^{c h}(\mathcal{L})^{V}$ of $\mathcal{L}^{V}$.

\footnotetext{
${ }^{7}$ In fact, by Remark in $2.8, \mathcal{L}_{\mathcal{C}}^{0}$ identifies with the Atiyah Lie* $R$-algebroid of infinitesimal symmetries of the Tate $R$-modules $\Theta_{R}$ or $\Omega_{R}$.
} 
Remark. The key ingredient of $\mathcal{O}$ - and $\mathcal{D}$-module theory in the usual finitedimensional setting is functoriality of the derived categories with respect to morphisms of varieties. The Clifford module picture permits to recover the pull-back functoriality for appropriately twisted derived categories (the $\mathbb{Z}$-grading of complexes should be labeled by the dimension $\mathbb{Z}$-torsor, and the derived category itself should be understood in an appropriate way). This construction is necessary in order to define the notion of $\mathcal{O}$ - or $\mathcal{D}$-module on orbit spaces such as the moduli space of (de Rham) local systems on the formal punctured disc. I hope to return to this subject in a joint work with Gaitsgory.

2.10. The weak $P B W$ theorem for topological chiral algebroids. Let $\left(R, \mathcal{L}, \mathcal{L}^{b}\right)$ be a topological chiral Lie algebroid where $R$ is reasonable. The morphisms $R \rightarrow U^{c h}(\mathcal{L})_{0}^{b}, \mathcal{L} \rightarrow \operatorname{gr}_{1} U^{c h}(\mathcal{L})^{b}$ yield then a morphism of topological coisson algebras (see (2.3.1))

$$
\operatorname{Sym}_{R}^{!} \mathcal{L} \rightarrow \widehat{\operatorname{gr}} U_{R}^{c h}(\mathcal{L})^{b}
$$

Theorem. If $\mathcal{L}$ is a flat $R$-module with respect to $\otimes !$, then $\operatorname{Sym} m_{R}^{!} \mathcal{L} \stackrel{\sim}{\rightarrow}$ gr. $U_{R}^{\text {ch }}(\mathcal{L})^{b}$. Thus the standard filtration satisfies the weak $P B W$ property.

Proof. We follow $[\mathrm{BD}]$ 3.9.13. Set $U^{c h}:=U_{R}^{c h}(\mathcal{L})^{b}$ and $U:=U_{R}(\mathcal{L})^{b}$.

(a) Suppose our chiral extension admits a rigidification, so we have a Lie* algebra $L$ acting on $R, \mathcal{L}=L_{R}=R \otimes ! L$, and $\mathcal{L}^{b}=L_{R}^{b}$ is the $L$-rigidified chiral extension.

Then $U$ is the enveloping algebra of $(L, R)$, i.e., the quotient of the free associative algebra generated by $L \oplus R$ modulo the relations saying that the map $L \rightarrow U$ is a morphism of Lie algebras and the map $R \rightarrow U$ is a morphism of associative algebras which commutes with the $L$-action (where $L$ acts on $U$ via $L \rightarrow U$ and the adjoint action). Our $U^{c h}$ is the corresponding chiral enveloping algebra which can be constructed as follows. Consider the topology on $U$ whose base is formed by all left ideals $U(P+I)$ where $P \subset L$ and $I \subset R$ are open subspaces. Shrinking $P, I$ if necessary, one can assume that $P$ is an open Lie subalgebra of $L$ and $I$ an open $P$-stable ideal of $R$. This topology satisfies the conditions from Corollary in 1.4 , hence $U^{\text {ch }}$ is the completion of $U$ with respect to this topology.

For $(P, I)$ as above the quotient $U / U(P+I)$ coincides with the $L$-module induced from the $P$-module $R / I$. Thus $\operatorname{gr}(U / U(P+I))=\operatorname{Sym}(L / P) \otimes R / I$ by the usual PBW theorem. Passing to the projective limit with respect to $(P, I)$, we see that the standard filtration satisfies the PBW property (actually, the strong one).

(b) Now let $\mathcal{L}$ be an arbitrary Lie* $R$-algebroid and $\mathcal{L}^{b}$ is chiral extension. Let $L$ be a copy of $\mathcal{L}^{b}$ considered as a mere Lie* algebra acting on $R$. Consider the corresponding Lie* $R$-algebroid $L_{R}=R \otimes ! L$ and its $L$-rigidified chiral extension $L_{R}^{b}$. We have an evident morphism of Lie* $R$-algebroids $L_{R} \rightarrow \mathcal{L}$ and its lifting to the chiral extensions $L_{R}^{b} \rightarrow \mathcal{L}^{b}$. The projection $L_{R}^{b} \rightarrow L_{R}$ identifies the kernels of those morphisms. Our $K$ is an $R$-module (in the $\otimes$ ! sense, as a submodule of $L_{R}$ ) equipped with a continuous $L$-action (the adjoint one).

Set $\tilde{L}_{R}:=\operatorname{C}$ one $\left(K \hookrightarrow L_{R}\right), \tilde{L}_{R}^{b}:=\operatorname{Cone}\left(K \hookrightarrow L_{R}^{b}\right)$. Then $L_{R}$ is naturally a DG Lie* $R$-algebroid, and $\tilde{L}_{R}^{b}$ is its chiral extension. These structures are uniquely 
defined by the condition that the embedding $L_{R} \rightarrow \tilde{L}_{R}$ is a morphism of Lie* $R$ algebroids, and that $L_{R}^{b} \rightarrow \tilde{L}_{R}^{b}$ is a morphism of chiral extensions. Therefore we have a DG chiral algebra $U_{R}^{c h}\left(\tilde{L}_{R}\right)^{b}$.

Set $\tilde{R}:=\operatorname{Sym}_{R}^{! \cdot}(K[1])$; this is a commutative! graded topological $R$-algebra whose component in degree $-i$ equals $\Lambda_{R}^{! i} K$. It carries a natural continuous $L$-action. So we have the (Z्Z-graded) Lie* $\tilde{R}$-algebroid $L_{\tilde{R}}$, its $L$-rigidified chiral extension $L_{\tilde{R}}^{b}$, and the corresponding chiral enveloping algebra $U_{\tilde{R}}^{c h}\left(L_{\tilde{R}}\right)^{b}$.

Lemma. There is a unique isomorphism of $\mathbb{Z}$-graded topological chiral algebras

$$
U_{R}^{c h}\left(\tilde{L}_{R}\right)^{b} \stackrel{\sim}{\rightarrow} U_{\tilde{R}}^{c h}\left(L_{\tilde{R}}\right)^{b}
$$

which identifies copies of $R$ and $L$ in the degree 0 components, and identifies $K \subset$ $\tilde{L}_{R}^{b}$ with $K \subset \tilde{R}$ in the components of degree -1 .

Proof. Both algebras are generated by $R, K$, and $L$ with same relations.

Isomorphism (2.10.2) is identifies the standard filtrations up to a shift by the grading: one has $U_{R}^{c h}\left(\tilde{L}_{R}\right)_{n}^{b a} \stackrel{\sim}{\rightarrow} U_{\tilde{R}}^{c h}\left(L_{\tilde{R}}\right)_{a+n}^{b a}$. By $($ a $), U_{\tilde{R}}^{c h}\left(L_{\tilde{R}}\right)^{b}$ satisfies the PBW property. So we have an isomorphism of graded DG chiral algebras

$$
\operatorname{Sym}_{R}^{!} \tilde{L}_{R} \stackrel{\sim}{\rightarrow} \operatorname{gr} . U_{R}^{c h}\left(\tilde{L}_{R}\right)^{b} .
$$

(c) Suppose that $\mathcal{L}$ is $R$-flat with respect to $\otimes$ !. Then the projection $\operatorname{Sym}_{R}^{!} \tilde{L}_{R} \rightarrow$ $\operatorname{Sym}_{R}^{!} \mathcal{L}$ is a quasi-isomorphism in the exact category $R \bmod !$. Therefore the differential $d$ on $U_{R}^{c h}\left(\tilde{L}_{R}\right)^{b}$ is strictly compatible with the standard filtration. Let $I_{n} \subset U_{R}^{c h}\left(\tilde{L}_{R}\right)_{n}^{b}$ be the closure of $K U_{R}^{c h}\left(\tilde{L}_{R}\right)_{n-1}^{b}$; then $I_{n}=I_{m} \cap U^{c h}\left(\tilde{L}_{R}\right)_{n}^{b}$ for any $m \geq n$, hence gr. $U_{R}^{c h}(\mathcal{L})^{b} \underset{\leftarrow}{\sim}$ gr. $U_{R}^{c h}\left(\tilde{L}_{R}\right)^{b} /$ gr. $I \stackrel{\sim}{\rightarrow} \operatorname{Sym}_{R} \mathcal{L}$, q.e.d.

Exercise. Suppose the topology of $R$ has a base formed by open reasonable ideals $I$ that satisfy the next property: The open Lie* $R$-subalgebroids $M \subset \mathcal{L}$ such that $M(I) \subset I, M \supset I \mathcal{L}$, and $\mathcal{L} / M$ is a flat $R / I$-module, form a base of the topology of $\mathcal{L} / \overline{I \mathcal{L}}$. Then the standard filtration on $U_{R}^{c h}(\mathcal{L})^{b}$ is a strong PBW filtration.

\section{REFERENCES}

[BD] A. Beilinson and V. Drinfeld, Chiral Algebras, AMS, Providence, RI, 2004.

[D] V. Drinfeld, Infinite-dimensional vector bundles in algebraic geometry: an introduction, The unity of mathematics, Progr. Math., vol. 244, Birkhäuser, Boston, MA, 2006, pp. 263-304.

[G] A. Grothendieck, Produits tensoriels topologiques et espaces nucléaires., Mem. AMS (1955), no. 16.

[MSV] F. Malikov, V. Schechtman, and A. Vaintrob, Chiral de Rham complex, Commun. Math. Phys. 204 (1999), 439-473.

[KV] M. Kapranov and E. Vasserot, Vertex algebras and the formal loop space, Publ. Math. IHES 100 (2004), 209-269.

[S] J.-P. Schneiders, Quasi-abelian categories and sheaves, Mém. Soc. Math. Fr. (1999), no. 76. 